Meta

Journal des traducteurs

Translators' Journal

\title{
Les incertitudes du traduire
}

\section{Lance Hewson}

Volume 61, numéro 1, mai 2016

Des zones d'incertitudes en traduction

URI : https://id.erudit.org/iderudit/1036980ar

DOI : https://doi.org/10.7202/1036980ar

Aller au sommaire du numéro

Éditeur(s)

Les Presses de l’Université de Montréal

ISSN

0026-0452 (imprimé)

1492-1421 (numérique)

Découvrir la revue

Citer cet article

Hewson, L. (2016). Les incertitudes du traduire. Meta, 61(1), 12-28.

https://doi.org/10.7202/1036980ar

\section{Résumé de l'article}

L'incertitude est une valeur fondamentale de la traduction. Pourtant, peu de traductologues s'y sont intéressés. La cartographie des incertitudes proposée dans cet article montre clairement qu'elles peuvent se manifester tout au long de l'opération traduisante. Plusieurs aspects de cette opération et les divers acteurs qui y participent sont examinés. On s'intéresse, tout d'abord, au rôle du donneur d'ouvrage. On se penche sur les aléas de l'interprétation du texte source, et en particulier sur le double regard du traducteur. La contribution d'autres acteurs est évoquée, en particulier le rôle de l'auteur, et les éventuelles ingérences postérieures à la phase de révision de la traduction. On s'intéresse, enfin, aux destinataires du texte traduit, en exploitant la distinction entre le lecteur modèle et le lecteur empirique. Après ce regard critique sur les phases en amont et en aval de l'acte traductif proprement dit, les incertitudes liées aux choix traductifs sont explorées. Quelques paragraphes sont consacrés aux certitudes du traducteur. Deux conclusions ressortent de ces réflexions : il est clair que la traductologie se doit d'intégrer l'incertitude au sein de ses préoccupations ; enfin, ce phénomène bien connu des professionnels devrait être considéré comme un atout qui permet au traducteur de rester en état de vigilance.
Ce document est protégé par la loi sur le droit d'auteur. L’utilisation des services d’Érudit (y compris la reproduction) est assujettie à sa politique d'utilisation que vous pouvez consulter en ligne.

https://apropos.erudit.org/fr/usagers/politique-dutilisation/ 


\title{
Les incertitudes du traduire
}

\author{
LANCE HEWSON \\ Université de Genève, Genève, Suisse* \\ Lance.Hewson@unige.ch
}

\section{RÉSUMÉ}

L'incertitude est une valeur fondamentale de la traduction. Pourtant, peu de traductologues s'y sont intéressés. La cartographie des incertitudes proposée dans cet article montre clairement qu'elles peuvent se manifester tout au long de l'opération traduisante. Plusieurs aspects de cette opération et les divers acteurs qui y participent sont examinés. On s'intéresse, tout d'abord, au rôle du donneur d'ouvrage. On se penche sur les aléas de l'interprétation du texte source, et en particulier sur le double regard du traducteur. La contribution d'autres acteurs est évoquée, en particulier le rôle de l'auteur, et les éventuelles ingérences postérieures à la phase de révision de la traduction. On s'intéresse, enfin, aux destinataires du texte traduit, en exploitant la distinction entre le lecteur modèle et le lecteur empirique. Après ce regard critique sur les phases en amont et en aval de l'acte traductif proprement dit, les incertitudes liées aux choix traductifs sont explorées. Quelques paragraphes sont consacrés aux certitudes du traducteur. Deux conclusions ressortent de ces réflexions: il est clair que la traductologie se doit d'intégrer l'incertitude au sein de ses préoccupations; enfin, ce phénomène bien connu des professionnels devrait être considéré comme un atout qui permet au traducteur de rester en état de vigilance.

\section{ABSTRACT}

Despite the fact that all translation necessarily involves a degree of uncertainty, translation theorists have show little interest in the phenomenon. The various types of uncertainty that may occur throughout the translation process are mapped out. Different aspects of the translation operation are examined, together with the various players involved, in particular the important role of the initiator, the more minor role of the author and the sometimes unpredictable decisions of the editor. The uncertainties that arise when interpreting the source text are investigated, as is the translator's split vision between source and target. The role of the reader is also evoked, using the distinction between the model reader and the empirical reader. Attention is then focused on the various uncertainties that the translator must face when exploring the paraphrastic possibilities of reformulation in the target language. The translator's certainties are also briefly examined. Two conclusions are drawn: the first concerns the need to devote more theoretical space to the question of uncertainty. The second underlines how this phenomenon - that professional translators have to deal with on a day to day basis - can be considered in a positive light, helping translators to remain alert during all the stages of their work.

\section{MOTS CLÉS/KEYWORDS}

cartographie, donneur d'ouvrage, interprétation du texte source, lecteurs modèle et empirique, créativité

mapping, initiator, interpretation of source text, model and empirical readers, creativity 


\section{Introduction}

L'incertitude est, curieusement, absente de la grande majorité des réflexions traductologiques. Le lecteur s'interrogera sans doute sur le choix de l'adverbe curieusement. En effet, dira-t-on, n'est-il pas normal d'écarter d'une réflexion théorique un état psychologique passager, subjectif et difficilement mesurable? Après tout, la traductologie vise à rendre compte de phénomènes bien plus larges, plus essentiels, qui concernent l'ensemble de l'opération traduisante. Enfin, pourrait-on conclure, le traductologue n'a aucun intérêt à mettre en exergue l'un des divers états qui caractérisent tout exercice d'écriture ou de réécriture. Pourtant, comme nous le verrons, il faudra donner raison à Alexander Künzli, qui place l'incertitude parmi «un ensemble de facteurs importants qui entrent en jeu dans la traduction au quotidien» (Künzli 2003: 8). Il s'agit, en effet, d'un phénomène qui, de notre point de vue, devrait trouver toute sa place au sein de la réflexion traductologique, et ce, pour deux raisons. La première tient à la nature même de la pratique traductive, dont on connaît la complexité et la difficulté. Certes, le traducteur, fort de son expérience professionnelle, a l'impression de contrôler un certain nombre de paramètres de cet exercice de réécriture. Il constate qu'il peut parfois s'inspirer de solutions de traduction qui, par le passé, ont été validées. Pourtant, il sait pertinemment qu'il ne pourra pas compter sur la plupart des «solutions» de reformulation qui lui viendront spontanément à l'esprit, car celles-ci ne seront pas en adéquation avec l'interprétation qu'il souhaite privilégier du texte source. Il doit, par conséquent, faire appel à une inventivité et à une créativité qui sont à mille lieues d'une réécriture prévisible et mécanique. S'ensuit un processus d'expérimentation qui a nécessairement sa part d'incertitude. Cette première raison est donc intimement liée au déroulement prévisible de l'acte traductif proprement dit. On conviendra, cependant, que le chercheur ne saurait se contenter de cette vision un peu naïve de la traduction qui, en se focalisant sur l'acte lui-même, gomme tout ce qui l'entoure. Par conséquent - et nous en venons ici à notre seconde raison -, il est nécessaire d'étudier la manière dont d'incertitude peut se manifester en amont et en aval de l'acte traductif pour avoir une vision d'ensemble de tous les aspects de l'opération de traduction. Pour ce faire, nous proposons d'établir une "cartographie» qui définit les divers «lieux» où les incertitudes sont susceptibles d'émerger et d'accompagner le traducteur dans l'exercice de sa profession.

L'exploration que nous proposons permettra de comprendre que les incertitudes du traduire proviennent de sources diverses, et qu'elles se ressentent tantôt de plein fouet, tantôt en sourdine. On nous objectera, avec raison, que tout en traduction n'est pas incertitude, et que le traducteur peut s'appuyer sur un certain nombre de certitudes. Nous nous emploierons toutefois à démontrer que ces dernières ne sont directement liées ni aux choix auxquels le traducteur est confronté ni aux décisions qu'il doit prendre; elles s'appuient, en revanche, sur une connaissance détaillée de l'ensemble des paramètres qui interviennent tout au long de l'opération traduisante. Nous constaterons, enfin, que l'incertitude n'est pas un état passager réservé à l'apprenti traducteur; elle accompagne le professionnel dans ses activités et, surtout, peut contribuer à l'efficacité de son travail. Nous pourrons ainsi conclure que les incertitudes constituent une facette inévitable, mais positive de l'expérience du traduire. 


\section{Cartographie des incertitudes}

Nous avons vu que l'objet de notre cartographie est d'examiner l'opération de traduction dans son ensemble, en y incorporant les phases en amont et en aval de l'acte de traduction proprement dit. L'action du donneur d'ouvrage, qui formule une commande de traduction, constitue le point de départ de la réflexion. Le deuxième volet de la cartographie, traitant de l'interprétation du texte source, se répartit en trois sous-parties: la problématique de la lecture-interprétation, la spécificité du double regard du traducteur, et l'auteur et son «vouloir-dire». Le troisième volet, intitulé "projections vers la cible», porte sur les incertitudes du skopos, du rôle de l'editor, et de l'encadrement du destinataire. La quatrième et dernière section concerne l'acte de traduction proprement dit. Elle prolonge la réflexion esquissée dans notre introduction sur les parties plus mécaniques et plus créatives du traduire, et a également pour objet la question de l'authenticité en traduction.

\subsection{Le donneur d'ordre: entre conscience et ignorance, action et inaction}

On pourrait naïvement imaginer que, dans le meilleur des mondes, le rôle du donneur d'ordre serait de faire en sorte que le traducteur puisse maîtriser ses incertitudes; on pourrait même espérer qu'il remplirait ce rôle d'office, sans qu'il soit besoin de lui demander quoi que ce soit. Or, même s'il est exact que certains donneurs d'ordre permettent aux traducteurs de travailler dans de bonnes conditions, force est de constater que, dans le monde réel de la traduction professionnelle, cet acteur incontournable de l'opération traduisante peut s'avérer être un obstacle, et non des moindres. Plusieurs cas de figure peuvent se présenter, selon la propension à l'action du commanditaire et selon la compréhension qu'il a de sa propre fonction.

Prenons tout d'abord le cas d'un donneur d'ordre inactif, qui ne réagit pas aux demandes d'informations et de clarifications qu'il reçoit du traducteur. Comment expliquer cette absence de réactivité? Certains donneurs d'ordre ignorent la nature de la traduction et les difficultés qui s'y associent. Ancrés dans leurs certitudes, ils ne donnent pas au traducteur le soutien dont il a besoin. On peut également imaginer d'autres raisons qui motivent cette inaction. Puisque le traducteur ne jouit pas du statut professionnel qu'il mérite (Pym, Grin et al. 2012), on peut concevoir un donneur d'ordre assez désinvolte, qui se désintéresse de tout ce qui concerne les tâches du traducteur. Un troisième cas de figure est celui d'un donneur d'ordre qui se trouve au milieu d'une chaîne de responsabilités. C'est le cas lorsqu'un traducteur sous-traite un mandat à un autre traducteur. Il peut arriver que ce traducteur devenu donneur d'ordre ne soit pas en mesure de répondre aux questions posées par le traducteur effectif, parce qu'il ne peut pas ou ne veut pas se renseigner auprès du commanditaire originel, ou parce que ce dernier refuse de fournir les informations demandées. Cette situation problématique n'est pas inhabituelle chez le traducteur pigiste: obligé de sous-traiter pour cause de surmenage, menacé par des échéances rapprochées, il ne veut surtout pas entendre parler de son collègue sous-traitant, qui doit s'exécuter du mieux qu'il peut. Et il pourra refuser de mettre le traducteur effectif en contact direct avec le demandeur, voire de faire remonter ses questions, par peur de se faire courtcircuiter ou d'avouer qu'il n'a pas fait le travail lui-même. Quelles que soient les causes de l'inaction du donneur d'ordre, on conviendra que l'absence d'informations et l'absence de clarifications qu'elle entraîne sont d'importantes sources d'incertitudes. 
Si les incertitudes liées à l'ignorance et à l'inaction du donneur d'ordre sont déjà considérables, celles qui tiennent à la conscience et à l'action de celui-ci sont autrement plus redoutables. Tout d'abord, une mise au point terminologique s'impose. Nous entendons ici par «conscience» non pas une quelconque compétence en matière de traduction, mais une idée précise du pouvoir et des droits dont jouit la personne qui chapeaute le travail de traduction et qui rémunère le traducteur une fois le travail terminé. Rappelons que le donneur d'ordre joue un rôle clé au début du mandat de traduction (il propose un cadre, une rémunération et un calendrier); qu'il peut demander à intervenir au cours de l'acte traductif; enfin, qu'il fait ce qu'il veut du produit fini. Nous avons déjà eu l'occasion d'insister sur l'autonomie d'action de cette instance, qui peut demander à une tierce personne, par ailleurs incompétente, de réviser la traduction fournie par le traducteur (Hewson 2013, 2015). Pire, le traducteur doit apprendre à vivre avec les lubies du donneur d'ordre, qui, profitant du pouvoir associé à sa position, peut exiger que le traducteur modifie sa façon habituelle de travailler, ou peut tout simplement en rejeter le résultat. Dans son ouvrage intitulé Kreatives Übersetzen, Kussmaul (2000: 46) note que le donneur d'ordre, qui n'a aucune compétence en traduction (tout en se croyant expert), se donne fréquemment la tâche d'évaluer lui-même la traduction fournie par le traducteur. Kussmaul cite également un exemple de Hönig (1995: 181), qui montre à quel point un donneur d'ordre peut entraver le travail du traducteur, et même refuser une traduction pour des raisons fondées sur une ignorance des réalités traductives.

Une dernière remarque pour conclure cette section: les aléas de la profession étant ce qu'ils sont, beaucoup de traducteurs ne peuvent pas se permettre de refuser des conditions de travail insatisfaisantes: ils doivent même accepter de nouvelles collaborations avec des donneurs d'ordre difficiles, voire non professionnels dans leur comportement.

\subsection{Les méandres de l'interprétation du texte source}

Les incertitudes présentées ci-dessus sont intimement liées à des comportements et à des prises de position adoptés par l'instance incontournable de chaque opération traduisante qu'est le donneur d'ordre. On peut également estimer que, dans certaines conditions, ces incertitudes sont pour ainsi dire inexistantes: si, par exemple, le traducteur est son propre commanditaire, ou si ce dernier donne des explications limpides et s'avère explicite dans sa démarche. Il convient, en revanche, d'insister sur les incertitudes qui découlent des difficultés d'interprétation du texte source. Nul besoin de rappeler qu'en principe, tout acte de traduction nécessite une étape préalable d'interprétation du texte source. Cette règle générale est, certes, à nuancer, comme l'a fait Umberto Eco, qui en exclut les traductions «médiocres de textes médiocres, commandées dans le seul but de gagner de l'argent sans perdre de temps » (Eco 2006: 290-291). On peut même aller plus loin en faisant valoir que toute traduction, quelle que soit la valeur du texte source, peut être qualifiée de «médiocre» si le traducteur n’y met pas le temps (et le talent, l'intérêt, l'énergie, etc.) nécessaire. Cependant, là n'est pas l'enjeu de la traductologie. Il est, par conséquent, important d'examiner la nature spécifique de l'acte interprétatif dans le cadre de l'opération traduisante. Pour ce faire, nous nous livrerons d'abord à quelques observations générales susceptibles de concerner tout texte source et les incertitudes qu'il recèle; nous 
examinerons par la suite les caractéristiques particulières de la lecture-interprétation dans le contexte spécifique de la traduction; nous évoquerons, enfin, l'importance toute relative de l'auteur du texte source et de son "vouloir-dire», dans l'intention d'écarter des réflexions traductologiques cette dernière notion, qui nous paraît bien floue.

\subsubsection{Quelques constantes concernant la lecture-interprétation du texte source}

Des difficultés d'interprétation guettent le traducteur au même titre que tout lecteur. Nous devons en effet, au risque d'énoncer une lapalissade, insister sur la manière dont un texte ne se «livre» pas au lecteur. Comme nous le rappelle Bruner (1991: 9), le lecteur n'attend pas que se manifeste une quelconque ambigüité pour se lancer dans l'acte d'interprétation, car c'est la narration elle-même qui stimule cette activité. Il est, d'ailleurs, toujours utile de rappeler que tout texte est potentiellement «difficile». Dans une étude empirique portant sur la prise de risque en traduction, Künzli (2004) met en évidence l'incertitude vécue par des traducteurs et des étudiants en traduction lorsqu'ils se retrouvent face à un texte pragmatique qui, à première vue, ne présente aucune difficulté d'interprétation (il s'agit du mode d'emploi d'un téléphone-télécopieur produit par France-Télécom). Il explique pourquoi une phrase d'apparence toute simple ( Insérez la petite fiche du bloc alimentation dans le connecteur (C) : ouvrez le capot avant et le capot arrière») (Künzli 2004: 35) donne du fil à retordre à tous ceux qui prennent part à l'exercice. Le lecteur-traducteur doit, en l'occurrence, interpréter le choix de ponctuation (les deux-points) dans le cadre de l'énoncé dans son ensemble. Grâce à l'emploi de protocoles de verbalisation, l'auteur décrit les incertitudes vécues par les participants en matière de compréhension, et, bien entendu, lorsqu'ils doivent restituer le texte en langue cible.

On peut, sans exagération aucune, avancer qu'il existe des millions de textes semblables, dont la rédaction quelque peu «lâche» ne permet pas une interprétation claire et en adéquation avec la finalité présumée du texte. Avant même d'aborder le texte littéraire, on peut passer en revue quelques-unes des raisons qui conduisent à ces difficultés. Une figure de style mal choisie, par exemple, peut brouiller la lecture. Un style elliptique peut ouvrir de multiples possibilités d'interprétation, remarque qui vaut aussi pour un terme ambigu, comme le souligne Eco (1999). On doit aussi prendre en considération le nombre de plus en plus important de documents rédigés directement dans une langue étrangère, dont l'anglais lingua franca est sans doute l'exemple le plus répandu. Nous avons déjà eu l'occasion de commenter certaines caractéristiques de ces textes, qui peuvent poser au traducteur des problèmes quasiment insolubles (Hewson 2013). Il est intéressant d'évoquer brièvement la différence entre des textes "natifs» présentant des difficultés de compréhension et les textes rédigés en anglais lingua franca. Pour les premiers, il est raisonnable de postuler que l'auteur exerce un certain contrôle (voire un contrôle certain) sur ce qu'il écrit. Si le résultat est jugé déficient, ce n'est pas forcément la maîtrise de la langue qui est en cause, mais plutôt l'écart entre le projet et sa réalisation. Froeliger décrit cette situation comme suit:

la distance peut être grande entre la pensée et sa transcription. Persuadé d'être compréhensible puisqu'il se comprend lui-même sans aucune difficulté, notre rédacteur initial ne le sera pas toujours pour ses lecteurs, et donc pour ses traducteurs. (Froeliger 2013:36) 
La situation concernant les textes rédigés en lingua franca est plus complexe, car, même si un auteur écrit très bien, il ne maîtrise pas toujours tous les paramètres de l'écriture, et il peut lui manquer quelques outils d'appréciation de nuance. Devant le texte non natif, par conséquent, l'incertitude du traducteur provient également de l'impossibilité de se fier entièrement aux choix linguistiques du texte source.

On peut estimer que les difficultés d'interprétation présentées par le texte littéraire sont en général d'une autre nature. Le regard du traducteur est, en principe, dirigé en même temps vers des grandeurs d'ordre différent: il scrute les difficultés posées sur le plan microstructurel, tout en restant attentif aux effets qui se dégagent sur le plan macrostructurel. Il n'est pas étonnant de constater que, dans bien des cas, c'est le niveau microstructurel qui préoccupe le traducteur. Il doit, en effet, rester attentif à une combinaison d'éléments de nature différente: jeux de focalisation, passage entre discours direct, indirect et indirect libre, utilisation de procédés rhétoriques et prosodiques, exploitation de sociolectes, et ainsi de suite. Cependant, le traducteur se doit de rester également attentif sur le plan macrostructurel, puisque si celui-ci n'est pas pris en compte, il court le risque de déformer ou de gommer les thématiques qui parcourent l'ensemble de l'œuvre et de privilégier une hétérogénéité dans ses choix traductifs (Hewson 2014). Il lui incombe, par ailleurs, de dégager les lignes d'une interprétation qui s'appuie sur l'ensemble des marqueurs textuels, et qui établit un rapport avec la «réalité» en adéquation avec la structure pragmatique du texte (Lecercle 1999: 33). Prenons pour exemple la première phrase de la nouvelle de Maupassant, «La Maison Tellier»:

(1) On allait là, chaque soir, vers onze heures, comme au café, simplement.

(Maupassant 1881/1974: 256 $)$

Le lecteur traducteur doit s'interroger sur les valeurs à accorder à plusieurs éléments: comment comprendre le pronom personnel «on», sachant que celui-ci est remplacé par «ils» dès la deuxième phrase de la nouvelle? Comment expliquer le choix de «là»? Comment interpréter «simplement»? Si ces premières questions portent surtout sur la dimension linguistique, il est clair que l'élément «comme au café» exige une interprétation culturelle. Nous verrons dans la section qui suit que le traitement de ces éléments, c'est-à-dire la lecture-interprétation faite dans l'optique de la traduction à venir, n'est pas directement assimilable à la lecture «simple» à laquelle on procède lorsqu'on aborde un document rédigé dans sa propre langue. Nous constaterons, en effet, que le traducteur porte sur tout texte un regard double.

\subsubsection{La spécificité du double regard du traducteur}

Nous avons déjà avancé l'idée que le traducteur n'est pas simplement un lecteur «comme les autres» (Hewson 1995). Même s'il possède parfaitement la langue source, son regard sur le texte source reste un regard externe, informé bien entendu, attentif sans doute, mais excentré par rapport à un lecteur qui appartient pleinement au monde linguistique et culturel de la langue source. Sa lecture du texte et son interprétation de celui-ci sont par ailleurs orientées par l'acte traductif à venir. Attentif aux différences fondamentales entre l'ancrage linguistique et culturel du texte qu'il découvre et le potentiel traductif du nouveau texte à façonner dans la langue cible, il en produit une interprétation qui est en adéquation avec les contraintes encyclopédiques de la source, tout en lorgnant, déjà, du côté de la langue-culture cible. Il faut 
bien insister sur un point: cette phase de lecture-interprétation en vue d'une traduction précède la traduction proprement dite. L'attention du traducteur est forcément dédoublée, car, au moment même où il mobilise les connaissances encyclopédiques qui permettent d'interpréter le texte, son attention est focalisée sur la source et, pourtant, ancrée dans la cible. Cet ancrage dans la culture cible braque l'attention sur les différences de représentation fondamentales et permet de constater des ambigüités et des zones d'ombre qui, pour le lecteur appartenant à la seule langue-culture source, restent invisibles. Cette «visibilité» s'opère sur deux plans. Sur le plan linguistique, la double attention du traducteur s'active dès qu'il se trouve en présence d'une spécificité de la langue source, une forme syntaxique ou un élément grammatical par exemple. Sur le plan de la «réalité» qui sous-tend le texte, il est conscient de tout ce qui ne cadre pas mécaniquement avec les représentations habituelles de la langue-culture cible. Si l'on revient à l'exemple de Maupassant cité ci-dessus, et en prenant l'anglais comme langue cible, il est raisonnable de postuler que, lors de la lecture du passage en vue d'une traduction, plusieurs éléments mobilisent l'attention du traducteur anglophone, à savoir la non-correspondance entre le pronom « on » et sa traduction directe, "one», la difficulté que pose l'écart culturel entre le rituel d'aller au café dans le monde francophone et un rituel à définir dans une partie du monde anglophone, la présence de cinq propositions sans conjonction de coordination, et, enfin, l'écart chronologique entre une histoire située dans les dernières années du dix-neuvième siècle et son potentiel de réexpression et de représentation dans notre monde moderne. Une seule phrase et tant d'incertitudes!

\subsubsection{L'auteur et son « vouloir-dire»}

Sans aller jusqu'à prétendre que le «vouloir-dire» de l'auteur est considéré par certains comme une réponse sans équivoque à toute incertitude en matière d'interprétation, on peut se réjouir de l'incertitude des traductologues contemporains à l'égard de cette notion somme toute douteuse. On avait, en effet, l'habitude d'évoquer ce «vouloir-dire» comme si on y avait un accès direct. Pour Lederer (1994: 35) par exemple, cet accès est possible, à condition que l'apprenant ait la volonté de comprendre le vouloir-dire et qu'il possède des connaissances adéquates. Comme si une lecture objective du texte source était possible, en dehors de tout filtrage par la subjectivité et le positionnement culturel du lecteur (Brisset 1998). Des études récentes mettent en cause cette vision un peu naïve. À cet effet, elles insistent sur le filtre que constitue le bagage linguistique et culturel du lecteur. Balacescu et Stefanink, par exemple, notent:

[1]e fait que le sens ainsi traduit est toujours fonction du vécu personnel du récepteur/ traducteur a pour conséquence qu'il est illusoire de croire pouvoir atteindre à l'objectivité en traduction, voire de retrouver le «vouloir dire de l'auteur» (comme le préconise par ex. L'École de Paris). (Balacescu et Stefanink 2005: 637)

Boase-Beier (2006: 33) explicite cette problématique, en précisant que ce n'est pas l'intention de l'auteur qui rend l'interprétation possible mais bien ce que le texte lui-même dit. Eco (1996) adopte une position analogue quand il déclare, dans une conférence portant sur l'auteur et ses interprètes, qu'un texte est une machine «conçue pour éliciter des interprétations», en précisant qu'une consultation de l'auteur n'est pas pertinente. Nous estimons, par conséquent, qu'il est vain de partir 
à la recherche du «vouloir-dire» de l'auteur afin de diminuer, voire d'éradiquer, le sentiment d'incertitude qui, nécessairement, accompagne tout acte de lecture-interprétation. Il convient, cependant, de nuancer ce rejet en apportant une précision qui permet de distinguer entre texte pragmatique et texte littéraire. Pour le premier type de texte, il n'est pas inhabituel pour le traducteur de consulter l'auteur du document, dans une démarche qui vise à réduire les ambigüités et à clarifier l'interprétation d'un texte dont l'accès est difficile ${ }^{2}$. Ce qui, dans ce cas, est demandé à l'auteur correspond à une reformulation qui se substitue au texte originel et qui, avec la bénédiction de ce dernier, fait foi. On voit bien que la forme de ce texte originel n'est pas, a priori, immuable et qu'elle est, pour ainsi dire, dissociable de son "sens». Tout l'intérêt du texte littéraire, en revanche, repose sur un alliage entre forme et fond, ce qui rend toute reformulation problématique, ou, plus exactement, inappropriée. On pourrait dire, pour conclure cette section, que, tandis qu'il existe en traduction pragmatique une lueur d'espoir à chercher du côté de l'auteur, le traducteur littéraire doit faire de ses incertitudes un atout... comme nous essayerons de le montrer dans la dernière partie de notre article.

\subsection{Projections vers la cible}

Notre objet dans cette section est de mettre en évidence les limites de la réflexion traductologique sur la cible et de souligner les problèmes qui, pour le traducteur en exercice, persistent dès lors qu'il réfléchit au devenir du texte cible qu'il construit. Il sera question, dans une première sous-section, de s'interroger sur les interventions de l'instance que Pergnier désigne par le nom d'editor. Viendra ensuite une présentation critique des travaux sur la finalité de la traduction, autrement dit son skopos. Enfin, il sera question d'examiner ce que l'on peut dire des destinataires de la traduction et, surtout, ce que l'on ne peut absolument pas dire de ces mêmes destinataires.

\subsubsection{Le rôle de l'editor}

Dans un article publié en 1990, Pergnier met en garde contre les éventuelles interventions de l'instance qu'il appelle l'editor. Il déplore notamment les détériorations qui peuvent survenir une fois l'étape de la révision passée, en soulignant que le traducteur n'a aucune prise sur de tels agissements. Il note que l'intervention de l'editor

se manifeste souvent par l'introduction de fioritures et de restructurations de phrases destinées à «faire joli» et à donner du piquant à la prose. Cette réécriture de certains passages se fait parfois au grand dam de la fidélité au sens. (Pergnier 1990: 219)

En outre, lorsque le travail d'editing est mené en collaboration avec l'auteur du texte source, il «tend à tirer le texte vers une littéralité que le traducteur avait soigneusement évitée» (Pergnier 1990: 219). Pergnier ne condamne pas toute intervention de ce type, mais souhaite attirer notre attention sur la menace qui plane sur la qualité des traductions, surtout lorsque les interventions décrites sont attribuables à des individus qui n'ont aucune compétence traductive.

Dans le cadre de notre cartographie des incertitudes, il importe de mettre en exergue les doutes que peut connaître tout traducteur qui sait (ou soupçonne) que son travail sera mis entre les mains d'un «expert» dont les critères de correction lui échappent. 


\subsubsection{Les incertitudes $d u$ skopos}

À première vue, la théorie du skopos semble apporter des réponses concrètes aux questions que le traducteur peut formuler à l'égard de la cible de sa traduction. Selon les travaux de Reiss et Vermeer (1984), quand on comprend la finalité d'une traduction, on peut déterminer les stratégies adéquates pour que le texte cible remplisse la fonction qui lui est dévolue au sein de la nouvelle culture. Cette approche a sans aucun doute des retombées didactiques intéressantes et mérite sa place au sein du cours de traduction (Nord 2005). Cependant, il faut bien souligner que cette théorie repose sur deux notions, celle de finalité et celle de fonction, qui, en pratique, s'avèrent relativement floues. On a beaucoup insisté sur le fait que la finalité du texte cible est souvent différente de celle du texte source. Certes, mais comment y accéder, sachant que beaucoup de cahiers des charges sont muets sur ce point? Comment, par ailleurs, souscrire à une théorie qui, en minorisant à l'extrême le texte source, pourrait justifier de "produire un texte dont le sens est radicalement contraire à celui de l'original» (Fontanet 2012: 321) ? Quand on estime, en outre, que «définir le skopos de la traduction n'est pas toujours évident» (Lavault-Olléon 2001: 69), on voit à quel point une idée d'apparence lumineuse peut être source de confusion et, partant, d'incertitudes. On peut, d'ailleurs, multiplier les critiques à l'égard de cette approche en s'appuyant sur les écrits de House (2001 : 245), qui aborde la théorie du skopos dans l'optique de l'évaluation de la qualité des traductions. Selon cette traductologue allemande, c'est la théorie elle-même qui est fautive, car la notion de fonction n'y est jamais explicitée, et encore moins rendue opérationnelle. Enfin, la plupart des théoriciens, dont Munday en 2008, estiment que cette théorie ne s'applique pas à la traduction littéraire.

\subsubsection{Ce destinataire qui nous échappe}

Si l'importance du donneur d'ordre est souvent minimisée dans les écrits théoriques, on ne peut pas en dire autant du destinataire, qui, présent dès les premiers écrits sur la traduction (Ballard 1992), se voit consacrer toute l'attention. Il est instructif, en effet, de recenser les diverses manières dont il est intégré dans la réflexion traductologique. Dès les années 1960, Eugene Nida fait appel au lecteur pour sa définition de l'«équivalence dynamique», avec son principe d'«effet équivalent». Ce qui intéresse en particulier le chercheur américain, c'est de produire une traduction qui fasse en sorte que le rapport entre message et destinataires en langue cible soit «substantiellement le même» (Nida 1964: 159) que celui qui s'établit entre message et destinataires en langue source. La «réponse similaire» devient par la suite l'une des quatre exigences de base qui permettent de juger de la réussite d'une traduction ${ }^{3}$. Ce positionnement a, certes, été largement critiqué plus tard, comme le note Munday (2008: 43-44); ce qu'il convient néanmoins de souligner, c'est la prise en compte du destinataire en tant qu'instance intervenant dans les calculs traductifs. D'autres travaux accordent une importance similaire au destinataire. En particulier, Holz-Mänttäri (1986) lui donne le statut d'actant dans le processus de communication.

Ces avancées outre-Atlantique et outre-Rhin s'observent plus tardivement dans la traductologie francophone, où l'intérêt porté au destinataire se manifeste moins souvent et de manière moins systématique. Bensimon, par exemple, convoque celuici pour expliquer un comportement de traduction, en l'occurrence l'adaptation, 
décrite comme «un processus involontaire, lié aux contraintes de la langue cible et de l'environnement socio-culturel du destinataire» (Bensimon 1990: ix). Berman adopte une position plus radicale: la prise en compte du lecteur est une démarche à proscrire, une manipulation qui est synonyme de traduction-trahison (Berman 1985: 85). D'autres chercheurs adoptent une démarche contrastive: ils s'intéressent aux connaissances prévisibles des lecteurs du texte source, qui sont comparées à celles, présumées, des lecteurs de la traduction. Ainsi, Jean-Pierre Richard consacre un article aux difficultés posées par l'ignorance culturelle que cultive l'auteur à l'égard du lecteur du texte source, et, partant, par la distance culturelle interne ainsi créée. Il écrit notamment:

[s]i la distance culturelle du texte source au texte cible est parfois surévaluée, voire imaginaire, la distance culturelle interne qui peut structurer le texte original est souvent sous-estimée, voire ignorée. Sans conséquence pour la traduction des textes porteurs d'une culture homogène, généralement dominante (ce qu’aux États-Unis on nomme mainstream), cette erreur d'appréciation risque d'invalider la traduction d'œuvres bâties précisément sur le caractère pluriel de leur polysystème culturel. (Richard 1998: 151)

Le lecteur est également exploité dans les arguments présentés en faveur de diverses stratégies de traduction, dont l'explicitation, l'implicitation, ou la simplification (Klaudy 2009), ou, au contraire, pour mieux critiquer de telles stratégies, à l'instar de Chevalier et Delport (1995), qui, lorsqu'ils critiquent les pratiques traductives constatées dans bon nombre de traductions de Madame Bovary, convoquent l'image de lecteurs lésés.

D’autres chercheurs s'intéressent à l'importance stratégique qu'ont les destinataires d'une traduction. C'est le cas d'Andrew Chesterman dans ses travaux sur les normes (1997). En effet, Chesterman mise sur la possibilité de définir une norme, appelée expectancy norm, qui découle des attentes des destinataires. L'objet de cette norme est à la fois de guider le traducteur dans ses choix (modulés, donc, en fonction des attentes des lecteurs) et de permettre des jugements évaluatifs sur des traductions publiées (cette fois-ci selon l'idée que le critique se fait de ces mêmes attentes). Lapplication de cette norme semble assez problématique, car elle exige une recherche empirique sur la problématique de la réception qui, pour l'instant, n'en est qu'à ses débuts (Brems et Ramos Pinto 2013). Le lecteur est également mobilisé par un autre type de norme, appelé accountability norm, qui, dans la classification de Chesterman, est l'une des trois normes professionnelles qui encadrent le processus de traduction proprement dit. Il s'agit ici de s'assurer que la démarche du traducteur est une démarche éthique, ce qui implique une loyauté envers tous les acteurs engagés dans le processus de traduction (le lecteur final bien entendu, mais aussi l'auteur du texte source et le donneur d'ordre). Chesterman souligne la responsabilité du traducteur, qui doit se positionner par rapport à des demandes différentes, parmi lesquelles figurent, encore une fois, les besoins supposés des destinataires.

On aura vu que, dans les approches esquissées ci-dessus, le destinataire est perçu de diverses manières. On imagine ses réactions, on se penche sur ses besoins, on cherche à établir ses attentes. Cependant, le traducteur n'a pas de véritable prise directe sur celui qu'Eco (1985) nomme le «lecteur empirique». D'où la distinction faite par le sémiologue italien entre ce dernier, qui désigne les «vrais» lecteurs qui lisent effectivement le texte, et le "lecteur modèle», défini comme un effet du texte prévu par l'auteur de celui-ci, «capable de coopérer à l'actualisation textuelle de la 
façon dont lui, l'auteur, le pensait et capable aussi d'agir interprétativement comme lui a agi générativement» (Eco 1985: 71). Lane-Mercier (2001) a montré comment l'analyse du positionnement des lecteurs modèles peut éclairer la démarche d'une équipe de recherche qui s'est donné pour tâche de traduire les sociolectes dans Hamlet de Faulkner. Il nous semble néanmoins important de souligner, comme le fait Koster (2002), qu'agir «interprétativement» n'est pas le pendant exact d'un agir "génératif», et que le traducteur en est conscient. On pourrait ajouter que la démarche décrite par Lane-Mercier est exceptionnelle, sans aucun doute bien éloignée des possibilités et des préoccupations plus matérielles des traducteurs professionnels, quelles que soient leurs spécialisations. Nous ne souhaitons pourtant pas affirmer que le professionnel ne construit aucune image du lecteur modèle, car beaucoup de décisions traductives en dépendent. Ce qui nous semble plus réaliste, c'est d'affirmer que l'image du lecteur est convoquée par le traducteur lorsque celui-ci en ressent le besoin, c'est-à-dire pour résoudre des problèmes ponctuels, sur le seul plan microstructurel. Cette affirmation découle de nos propres observations de textes traduits, qui révèlent une hétérogénéité macrostructurelle qui peut surprendre (Hewson 2011, 2014). S’il n'en ressent pas le besoin, le traducteur se contente d'une idée un peu floue, c'est-à-dire non travaillée, du lecteur modèle. On peut, par ailleurs, étendre cette réflexion aux positions théoriques sur les «stratégies» du traducteur, qui, pourrait-on dire, sont davantage présentes dans les écrits des traductologues que dans les habitudes de bon nombre de praticiens.

Les observations ci-dessus permettent de conclure que le traducteur est très limité dans ses démarches focalisées sur ses futurs destinataires. Il peut, certes, travailler sur l'image du lecteur modèle; cependant, les lecteurs empiriques représentent une grande inconnue. Par conséquent, le traducteur ne peut s'affranchir de l'incertitude qui accompagne toute réflexion sur la destination d'une traduction. Il reste à voir quel est l'éventuel bénéfice que peut tirer le professionnel de cette situation, à première vue très inconfortable.

\subsection{Regards sur l'acte de traduction}

Nous souhaitons clore cette section en invoquant, enfin, la traduction proprement dite. Il était important, dans la perspective de notre cartographie, de passer en revue l'opération traduisante dans son ensemble, avant d'en arriver à l'activité du traduire, car l'examen des facteurs en amont et en aval de celle-ci révèle que la tâche de traduction n'est jamais une simple opération sur des langues et leurs cultures, mais une activité qui, paradoxalement, est fortement contrainte par des éléments intérieurs (les langues-cultures) et extérieurs (les facteurs évoqués en amont et en aval), tout en restant ouverte à la créativité. Il ne sera plus question, dans cette section, de ces éléments extérieurs, qui, néanmoins exercent leur influence en arrière-plan. Deux aspects de l'acte traductif seront examinés dans l'optique de l'incertitude: la marge de manœuvre entre mécanicité et créativité et la question de l'authenticité.

\subsubsection{Entre mécanicité et créativité}

Le traducteur expérimenté ne cherche pas, à tout moment, à explorer les nombreuses possibilités paraphrastiques qu'il pourrait exploiter. L'efficacité exige qu'il fasse confiance à son expérience, quand celle-ci lui permet de choisir la «solution» qui, 
par le passé, a déjà fait ses preuves. À la suite de Levý (1963/2011) et pour mieux décrire cette démarche, nous avons parlé de traduction «mécanique» (Hewson 2006). Nous avons également, dans la publication précitée et dans une publication ultérieure (Hewson 2012), exploré les limites d'une démarche créative. Entre ces deux extrêmes, à savoir, d'une part, le choix de la solution qui semble s'imposer d'elle-même et, d'autre part, la recherche d'une traduction créative, se trouve la zone où le traducteur se contente de passer en revue les possibilités de traduction standard, c'est-à-dire normalement prévisibles, sans entamer une démarche consciente vers la créativité ${ }^{4}$. Ce découpage de l'activité traduisante en trois mouvements possibles, dont l'actualisation varie en fonction, notamment, de l'expérience du traducteur et de la difficulté du texte proposé, permet de mieux comprendre l'émergence ponctuelle de l'incertitude au cours de l'acte traductif. En effet, lorsqu'on peut se contenter d'une activité de traduction mécanique, le degré d'incertitude est proche de zéro, puisque la solution, déjà testée, est considérée comme suffisante. Si l'on constate que la solution mécanique ne fonctionne pas, on se tourne vers les solutions standards, dont l'exploration s'accompagne d'une incertitude qui reste toute relative, car le traducteur reste dans sa zone de confort. Si, en revanche, et à la suite de l'échec de ses premières démarches, il doit quitter cette zone de confort, il se retrouve devant une incertitude maximale, celle qui est associée aux solutions non prévisibles qui, elles, relèvent de la créativité. À partir de cet instant, le traducteur est privé de ses repères habituels et s'interroge sur la pertinence de ses choix en tenant compte d'un certain nombre de paramètres tant externes qu'internes.

Il est bien entendu difficile de démontrer empiriquement que ces degrés d'incertitude correspondent au quotidien du traducteur. On peut néanmoins affirmer qu'un modèle de ce type permet d'établir une corrélation entre l'activité plus ou moins consciente menée par le traducteur et l'état psychique qui l'accompagne. Si d'autres paramètres concernant le traducteur doivent être pris en compte, on peut néanmoins retenir l'idée d'une incertitude variable, dont l'intensité est relative à ce type de démarche.

\subsection{2. À la recherche de l'authenticité}

La question de l'authenticité, ou de l'appartenance «réelle» à la langue cible, se pose de deux manières. Le traducteur qui travaille uniquement à partir d'une langue étrangère vers sa langue maternelle doit définir les limites de ce qui est acceptable dans sa langue et déterminer jusqu'où il peut excentrer celle-ci afin de produire l'effet voulu. Il ne suffit pas de se référer à la vieille dichotomie entre sourciers et ciblistes (Ladmiral 1986), car celle-ci, simplificatrice, ramène le débat à opposer deux types de comportements linguistiques (Hewson 2004). Même si la motivation de la démarche du traducteur trouve toujours son origine dans le texte source et dans l'interprétation qu'il a de celui-ci, il convient de se placer résolument du côté de la langue cible afin d'étudier ce qui est possible. La question qui se pose et qui est source d'incertitudes, même pour le traducteur très expérimenté, est celle-ci: comment définir ce qui est possible ou, plus simplement, quelle est la référence pour déterminer ce qui est possible? Autrement dit, quelle serait la norme de «bonne écriture», comment juger si tel ou tel énoncé est «conforme au génie de la langue» (Authier et Meunier 1972)? La réponse à cette question est des plus simples: il ne peut pas y avoir de norme qui écarterait définitivement toute expérimentation sur la langue. En 
l'absence de normes absolues, le traducteur doit accepter de prendre des risques. C'est ce qu'a fait, par exemple, Geoffrey Wall dans sa traduction de Madame Bovary ${ }^{5}$, en particulier lorsqu'il travaille l'agencement syntaxique en anglais (Hewson 2011). Si ce résultat est jugé positivement, d'autres ne le sont pas, comme le fait remarquer Bennett (2013: 180-182) dans son commentaire sur une traduction anglaise de Foucault ${ }^{6}$, qui, adoptant une démarche très littérale, est non seulement difficile à comprendre mais aussi à la limite du grammaticalement acceptable.

Il en va tout autrement pour celui ou celle qui traduit à partir de sa langue maternelle vers une langue étrangère. Cette pratique se fait de plus en plus fréquente dans bien des pays (Taviano 2013) et peut concerner autant les textes pragmatiques que les textes littéraires (Pokorn 2005). L'incertitude principale du traducteur qui travaille vers la langue étrangère concerne effectivement l'authenticité de la langue produite. Si l'on exclut de l'analyse toutes les «traductions» qui circulent sur l'Internet, manifestement produites par des personnes qui n'ont de traducteur que le nom (Hewson 2012), on est surtout frappé par le nombre de traductions qui sont, certes, correctes sur le plan grammatical, mais qui ne sont en aucun cas assimilables à ce qu'un professionnel travaillant vers sa langue maternelle aurait produit (Hewson 2013 : 265-269). Comment, en effet, être absolument certain que le nouveau texte est conforme à tous points de vue? Le contrôle que l'on exerce sur sa production en langue étrangère est tout à fait incomparable à celui que l'on exerce sur sa langue maternelle: pour ce traducteur, il existe, par conséquent, un degré supplémentaire d'incertitude linguistique et culturelle.

\section{Entre certitudes et incertitudes}

Après avoir exposé les incertitudes liées aux activités professionnelles du traducteur, il convient de voir s'il est possible d'affirmer que le professionnel peut également, dans l'exercice de son métier, compter sur des certitudes. À l'évidence, notre cartographie ne leur laisse pas beaucoup de place: nous avons vu que l'incertitude peut être présente à tout moment tout au long de l'opération traduisante. Devrait-on donc postuler que la certitude est un état inconnu du traducteur? Si la réponse à cette question est certainement négative, elle exige d'être nuancée. Pour ce faire, nous allons nous interroger sur la manière dont le parcours de l'apprenti traducteur peut permettre de trouver un juste équilibre entre deux positions extrêmes, dont l'une serait un état de certitude naïf et l'autre un vécu professionnel miné par des incertitudes de toutes sortes.

On peut concevoir l'apprentissage comme un parcours dont le départ est constitué d'un certain nombre de certitudes (pour relatives qu'elles soient), suivi d'une étape intermédiaire caractérisée par la montée en puissance des incertitudes et l'évanouissement des certitudes, pour prendre fin une fois que les exigences de la profession (et son lot inévitable d'incertitudes) auront été maîtrisées. Ces trois étapes méritent chacune un commentaire.

Comment peut-on parler de certitudes au début d'un apprentissage? Ce que nous visons ici, c'est l'ignorance du débutant qui, fier de ses connaissances en langues et de ses prouesses dans la manipulation de dictionnaires bilingues, croit avoir compris l'essence même du traduire. En effet, c'est lors des premiers cours de traduction que l'étudiant constate que ses performances ne correspondent absolument pas à ce que 
demande l'enseignant; il doit bientôt admettre que les certitudes qu'il croyait avoir n'étaient que des chimères.

L'étape suivante peut s'avérer délicate. Un enseignant qui se contenterait de brandir la «solution», sans donner les explications et les assurances qui s'imposent, risquerait de déstabiliser l'étudiant, comme le souligne Ballard:

Il ne s'agit pas de nier l'utilité de donner une version finale de qualité mais de constater qu'elle peut avoir à la fois un effet rassurant et paralysant. L'étudiant d'une part attend en toute sécurité le beau modèle qu'on va lui donner (LA SOLUTION, comme si un texte ne pouvait avoir qu'une traduction en langue étrangère), et d'un autre côté les qualités esthétiques de ce produit fini risquent d'en faire un objet trop lointain et presque inaccessible qui découragerait les efforts pour en approcher. (Ballard 1980:13)

En effet, lorsque l'étudiant comprend que sa propre traduction, produite à la suite d'un effort certain, est considérée comme fautive et insuffisante, il a besoin de pouvoir relativiser l'exercice et de comprendre qu'erreurs et maladresses font partie du parcours normal de l'apprenant. La responsabilité de l'enseignant est engagée, car, tout en encourageant une mise en question des certitudes, il lui incombe de travailler sur l'acquisition de compétences en vue d'acquérir la confiance nécessaire pour gérer les incertitudes naissantes. L'aide de l'enseignant peut être précieuse, surtout s'il est prêt à faire état de ses propres incertitudes, comme l'indique Künzli (2004: 47).

La dernière étape envisagée correspond à une prise de conscience des enjeux de la traduction, à une appréciation de l'importance et du rôle de tous les actants engagés dans l'opération traduisante (lato sensu), et à une maîtrise, hésitante au début mais montant en puissance, des compétences pertinentes. La fin de ce parcours correspond, par conséquent, à des certitudes concernant la nature du traduire et les démarches qui s'imposent. Il va de soi que les incertitudes ne sont pas estompées ou annulées. Au contraire, elles gagnent en visibilité et, petit à petit, se transforment en interrogations fécondes, qui renforcent la vigilance du traducteur et lui permettent de travailler avec davantage d'assurance. Autrement dit, une fois reconnues pour ce qu'elles sont, les incertitudes deviennent l'un des atouts à la disposition du traducteur, si bien qu'il convient, en conclusion, d'en faire l'éloge.

\section{Conclusion: les bienfaits de l'incertitude}

Notre cartographie nous a confirmé dans notre conviction que l'incertitude n'est pas un simple état passager, vécu occasionnellement par le traducteur lorsqu'il est en phase de réexpression, mais une valeur fondamentale associée à toute l'opération traduisante, qui se manifeste dès la commande de traduction et, dans la perspective des éventuelles interventions de l'editor, se perpétue même après la livraison du texte traduit. Notre interrogation concernant la place de l'incertitude dans les écrits traductologiques nous semble désormais légitime. Si quelques auteurs déjà évoqués, notamment Froeliger (2013) et Künzli $(2003,2004)$, ont déjà voulu placer l'incertitude dans leurs réflexions, force est de constater que, dans la majorité des écrits traductologiques, les chercheurs ne s'intéressent nullement à ce phénomène. Nous avons déjà essayé d'expliquer cet état de fait dans notre introduction, qui résulte certainement d'une perception très partielle, ou, pourrait-on dire, d'une non-perception, de l'importance de cet état. La traductologie devrait, à notre avis, intégrer l'incertitude au sein de ses préoccupations afin de mieux explorer les différents lieux 
de ses manifestations et de mieux comprendre le rôle qu'elle joue dans les processus traductifs.

Il est intéressant, en guise de conclusion, mettre en regard les certitudes du grand public concernant la traduction et les incertitudes du traducteur que nous avons documentées dans notre deuxième partie. L'accès à Internet avec son offre de traductions automatiques a eu comme effet de rendre la traduction instantanément disponible et, partant, banale. On ne se pose guère de questions sur la justesse de la traduction proposée par un logiciel, et encore moins sur sa qualité. Pour les Internautes, la traduction se résume à quelques clics. Nous avons vu que le traducteur professionnel ne partage pas cette certitude: il profite, certes, des avancées des technologies de la traduction, il se sert sans doute de temps à autre de la traduction automatique, mais il reste vigilant à l'égard des résultats obtenus. Il a acquis la certitude de son incertitude, et sait qu'il ne peut jamais baisser la garde. Même pendant ces moments de facilité, lorsque la traduction semble s'écrire presque toute seule, il sait qu'il doit rester en éveil, qu'il doit vérifier que toutes les conditions qu'il s'est imposées sont remplies. C'est l'incertitude qui est le moteur de son éveil, qui l'encourage dans son désir de produire la meilleure traduction possible.

\section{REMERCIEMENTS}

Nous souhaitons remercier Mathilde Fontanet de sa relecture attentive de cet article et de ses propositions d'amélioration. Nous souhaitons également remercier les deux lecteurs anonymes de leurs propositions d'amélioration.

\section{NOTES}

* Faculté de traduction et d'interprétation.

1. Maupassant, Guy de (1881/1974): La Maison Tellier. In: Contes et Nouvelles 1. Paris: Gallimard.

2. Froeliger (2013:49) souligne que le traducteur ne peut pas toujours compter sur la possibilité de joindre son auteur.

3. Les trois autres critères exigent que le texte cible fasse sens, qu'il transmette l'esprit et la manière de l'original, et que son expression soit naturelle et facile (Nida 1964: 164).

4. Cette vision n'est pas partagée par tous les traductologues. Ballard (1997), par exemple, estime que tout acte de traduction est en soi créatif.

5. Flaubert, Gustave (1857/1992). Madame Bovary. Traduit du français par Geoffrey Wall. Harmondsworth: Penguin Books.

6. Foucault, Michel (1966/2002): The Archaeology of Knowledge. Traduit du français par Alan M. SHERIDAN SMITH. Londres: Routledge.

\section{RÉFÉRENCES}

Authier, Jacqueline et Meunier, André (1972): Norme, grammaticalité et niveaux de langue. Langue Française. 16:49-62.

Balacescu, Iona et Stefanink, Bernd (2005): Défense et illustration de l'approche herméneutique en traduction. Meta. 50(2):634-642.

Ballard, Michel (1980): La traduction de l'anglais. Théorie et Pratique. Exercices de Morphosyntaxe. Lille: Presses universitaires de Lille III.

Ballard, Michel (1992): De Cicéron à Benjamin. Traducteurs, traductions, réflexions. Lille: Presses universitaires de Lille.

Ballard, Michel (1997): Créativité et traduction. Target. 9(1):85-110.

BennetT, Karen (2013) : English as a Lingua Franca in Academia. The Interpreter and Translator Trainer. 7(2): 169-193. 
Bensimon, Paul (1990): Présentation. Palimpsestes. 3:ix-xiii.

Berman, Antoine (1985): La traduction et la lettre - ou l'auberge du lointain. In: Antoine Berman, Gérard Granel et Annick Jaulin, dir. Les tours de Babel: essais sur la traduction. Mauvezin: Trans-Euro-Repress, 31-150.

BoAse-Beier, Jean (2006): Stylistic Approaches to Translation. Manchester: St. Jerome.

Brems, Elke et Ramos Pinto, Sara (2013): Reception and translation In: Yves Gambier et Luc van Doorslaer. Handbook of Translation Studies. Volume 4. Amsterdam/Philadelphie: John Benjamins, 142-147.

Brisset, Annie (1998): L'identité culturelle de la traduction. En réponse à Antoine Berman. Palimpsestes. 11:31-51.

BRUner, Jerome (1991): The Narrative Construction of Reality. Critical Enquiry. 18(1):1-21.

Chesterman, Andrew (1997): Memes of Translation. Amsterdam/Philadelphie: John Benjamins.

Chevalier, Jean-Claude et Delport, Marie-France (1995): L'horlogerie de Saint Jérôme. Paris: L'Harmattan.

Eco, Umberto (1979/1985): Lector in fabula: le rôle du lecteur ou la coopération interprétative dans les textes narratifs. Traduit de l'italien par Myriem Bouzaher. Paris: Grasset.

Eco, Umberto (1996): The Author and his Interpreters. Communication présentée à The Italian Academy for Advanced Studies in America, Columbia University, New York. Consulté le 23 mars 2015, <http://umbertoecoreaders.blogspot.ca/2007/11/author-and-his-interpreters. html $>$.

Eco, Umberto (1997/1999): Kant et l'ornithorynque. Traduit de l'italien par Julien Gayrard. Paris: Grasset.

Eco, Umberto (2003/2006): Dire presque la même chose. Expériences de traduction. Traduit de l'italien par Myriem Bouzaher. Paris: Grasset.

Fontanet, Mathilde (2012): Affinités respectives de l'anglais et du français en matière d'argumentation: étude comparative à l'usage du traducteur. Thèse de doctorat non publiée. Genève: Université de Genève. Consultée le 23 mars 2015, <http://archive-ouverte.unige. ch/unige:21615>.

Froeliger, Nicolas (2013): Les noces de l'analogique et du numérique. Paris: Les Belles Lettres.

Hewson, Lance (1995): Images du lecteur. Palimpsestes. 9:151-164.

Hewson, Lance (2004): Sourcistes et cibliers. In: Michel Ballard et Lance Hewson, dir. Correct/Incorrect, Arras: Artois Presses Université, 123-134.

Hewson, Lance (2006): The Vexed Question of Creativity in Translation. Palimpsestes. Hors série:53-63.

Hewson, Lance (2011): An Approach to Translation Criticism. Emma and Madame Bovary in Translation. Amsterdam/Philadelphie: John Benjamins.

Hewson, Lance (2012): Traduire: les limites de la créativité. In: Annick Ettuin et Fabien PiLlet, dir. Les mouvements de la traduction. Genève: MétisPresses, 113-128.

Hewson, Lance (2013): Is English as a Lingua Franca Translation's Defining Moment? The Interpreter and Translator Trainer. 7(2):257-277.

Hewson, Lance (2014): Baba, bouillie, brouet: les dangers de l'hybridité. Atelier de traduction. 22:23-34.

Hewson, Lance (2015): Auscultation du grand public. Parallèles. 27:10-19.

Holz-MäntтÄRI, Justa (1986): Translatorisches Handeln: Theorie und Methode. Helsinki: Suomalainen tiedeakatemia.

HöNIG, Hans (1995): Konstruktives Übersetzen. Tübingen: Stauffenburg Verlag.

House, Juliane (2001): Translation Quality Assessment: Linguistic Description versus Social Evaluation. Meta. 46(2):243-257.

Klaudy, Kinga (1998/2009): Explicitation. In: Mona Baker et Gabriela Saldanha, dir. Routledge Encyclopedia of Translation Studies. Londres/New York: Routledge, 104-108.

Koster, Cees (2002): The translator in between texts: on the textual presence of the translator as an issue in the methodology of comparative translator description. In: Alessandra 
Riccardi, dir. Translation Studies. Perspectives on an Emerging Discipline. Cambridge: Cambridge University Press, 24-37.

KüNZLI, Alexander (2003): Quelques stratégies et principes en traduction technique françaisallemand et français-suédois. Forskningsrapporter/Cahiers de la recherche 21. Stockholm: Stockholms universitet.

KüNZLI, Alexander (2004): Risk taking: trainee translators vs professional translators. Jostrans, The Journal of Specialised Translation. 2:34-49.

Kussmaul, Paul (2000): Kreatives Übersetzen. Tübingen: Stauffenburg Verlag.

Ladmiral, Jean-René (1986): Sourciers et ciblistes. Revue d'esthétique. 12:33-42.

LANE-Mercier, Gillian (2001) : Entre l'Étranger et le Propre: le travail sur la lettre et le problème du lecteur. TTR. 14(2):83-95.

LAVAult-OllÉon, Élisabeth (2001): La théorie du skopos en traduction littéraire et pragmatique: éclairages et contradictions. In: Michel BAndry et Jean-Marie Maguin, dir. La Contradiction, actes du congrès de la Société des anglicistes de l'enseignement supérieur. Montpelier: Publications Université Paul Valéry, 65-84.

LeCERCLe, Jean-Jacques (1999): Interpretation as Pragmatics. Londres: Macmillan.

LEDERER, Marianne (1994): La traduction aujourd'hui. Paris: Hachette.

LevÝ, JiŘí (1963/2011): The Art of Translation. Traduit du tchèque par Patrick Corness. Amsterdam/Philadelphie: John Benjamins.

Munday, Jeremy (2008): Introducing Translation Studies: Theories and Applications. Londres/ New York: Routledge.

NidA, Eugene A. (1964): Towards a Science of Translating: With Special Reference to Principles and Procedures Involved in Bible Translating. Leiden: E. J. Brill.

Nord, Christiane (1988/2005): Text Analysis in Translation: Theory, Methodology and Didactic Application of a Model for Translation-Oriented Text Analysis. Traduit de l'allemand par Christiane Nord et Penelope Sparrow. Amsterdam: Rodopi.

Pergnier, Maurice (1990): Comment dénaturer une traduction. Meta. 35(1):219-225.

PoкоRn, Nike K. (2005): Challenging the Traditional Axioms. Amsterdam/Philadelphie: John Benjamins.

Pym, Anthony, Grin, François, Sfreddo, Claudio et Chan, Andy (2013): The Status of the Translation Profession in the European Union. Bruxelles: Commission européenne. Consulté le 3 avril 2015, <http://ec.europa.eu/dgs/translation/publications/studies/translation_ profession_en.pdf $>$.

ReIss, Katherina et Vermeer, Hans J. (1984): Grundlegung einer allgemeinen Translationstheorie. Tübingen: Niemeyer.

Richard, Jean-Pierre (1998): Traduire l'ignorance culturelle. Palimpsestes. 11:151-160.

Taviano, Stefania (2013): English as a Lingua Franca and Translation. Implications for Translator and Interpreter Education. The Interpreter and Translator Trainer. 7(2):155-167. 